\title{
La investigación proyectual como estrategia didáctica en el proyecto del Taller de Diseño Arquitectónico
}

\author{
Project research as a didactic strategy in the Architectural Design Workshop \\ Project
}

César Fortunato Martínez-Vitor

Universidad Nacional del Centro del Perú. Huancayo (Perú)

Facultad de Arquitectura

Martínez-Vitor, C. F. (202I). La investigación proyectual como estrategia didáctica en el proyecto del Taller de Diseño Arquitectónico. Revista de Arquitectura (Bogotá), 23(2), 58-70. https://doi.org/10.14718/ RevArg.2021.3294
Arquitecto, Universidad Nacional del Centro del Perú. Huancayo, Junín (Perú).
Magister Scientiae en Gestión Ambiental y Desarrollo Sostenible, Universidad Nacional del Centro del Perú. Huancayo, Junín (Perú).
Doctor en Medio Ambiente y Desarrollo Sostenible, Universidad Nacional Federico Villareal. Lima (Perú).
https://scholar.google.es/citations?user=Jdfa9_UAAAAJ\&hl=es (iD) orcid.org/0000-000I-7I59-7973
@cesar_vitor@hotmail.com

\section{Resumen}

En la búsqueda de mejores estrategias didácticas en la enseñanza aprendizaje del estudiante de arquitectura, la aplicación de la investigación proyectual como estrategia didáctica influyó positivamente en el proyecto del Taller de Diseño Arquitectónico del décimo semestre 20I7-I de la Universidad Nacional del Centro del Perú, logrado a través de la investigación explicativa, cuasi experimental. Se emplearon métodos del planeamiento experimental, y para la evaluación del aprendizaje por competencias, se utilizó la rúbrica analítica. El análisis correspondiente se hizo mediante la estadística inferencial de las medianas de dos poblaciones apareadas del antes y el después, mediante la prueba de hipótesis no paramétrica de Wilcoxon. Los resultados indican que los conocimientos previos sobre investigación proyectual no fueron consistentes en la unidad I (etapa de entrada y adecuación), mientras que en la etapa de seguimiento y término sí existen diferencias significativas positivas en la evaluación de las unidades I y 3 , y 2 y 3 . Lo anterior demuestra que la aplicación de la investigación proyectual se ha fijado en los procedimientos del Taller de Diseño Arquitectónico, y cabe concluir que, ejercitada adecuadamente, la aplicación de la investigación proyectual como estrategia didáctica es parte relevante de la enseñanza-aprendizaje del estudiante de arquitectura.

Palabras clave: creatividad; enseñanza en la arquitectura; enseñanza profesional; escuela experimental; escuela profesional

\section{Abstract}

In the search for better didactic strategies in the teaching-learning process of the architecture student, the application of project research, as a didactic strategy, positively influenced the project of the Architectural Design Workshop of the 10th semester 2017-I at the Universidad Nacional del Centro del Perú, achieved through explanatory, quasiexperimental research. Methods of experimental planning were employed, and for the evaluation of learning by competencies, the analytical rubric was used. The corresponding analysis was made through inferential statistics of the medians of two paired populations, before and after, using the Wilcoxon non-parametric hypothesis test. The results indicate that prior knowledge on project research was not consistent in unit I (entry and adaptation stage), while in the follow-up and ending stage there are significant positive differences in the evaluation of units I and 3 , and 2 and 3 . The above shows that the application of project research has been set in the procedures of the Architectural Design Workshop, and it can be concluded that the application of project research, as a didactic strategy, properly exercised, is a relevant part in the teaching-learning process of the architecture student.

Keywords: architecture education; creativity; experimental schools; professional teaching; vocational colleges 


\section{Introducción}

Este artículo se presenta como resultado de la tesis final de maestría titulada Investigación proyectual como estrategia didáctica en el proyecto del Taller de Diseño Arquitectónico, adjunta a la línea de investigación Métodos Pedagógicos, en la Facultad de Arquitectura de la Universidad Nacional del Centro del Perú.

La investigación en la educación superior es bastante prolija en cuanto a su conocer, su entender y su aplicación del lenguaje, al igual que en cuanto a su enfoque científico a la solución de sus problemas. Actualmente, todas las disciplinas científicas asociadas a las especialidades profesionales $-\mathrm{y}$ sobre todo, la actividad dedicada a la formación superior- desarrollan su investigación para crear su propio lenguaje y su propio enfoque científico, tal cual es el caso de la formación del arquitecto. Como parte de la solución a la formación del arquitecto, planteo la investigación proyectual como estrategia didáctica en el proyecto del Taller de Diseño Arquitectónico.

En el taller de formación del arquitecto existen dos grandes fases: la primera es la información y la segunda es la decisión. Estas se resuelven según las características metodológicas del docente, que asume uno o más de los procesos: 1) deductivo, 2) inductivo o 3) abductivo. Sea cual sea el proceso asumido, ambas fases requieren una serie de acciones encaminadas a un fin determinado, definido como estrategia. La estrategia de la presente investigación se sustentó con los postulados y las bases teóricas y pedagógicas del constructivismo de Ferreiro (2007), que indicaron, a su vez, las estrategias del paradigma constructivista. Para el desarrollo de la experiencia proyectual, aplicada al taller investigado, se asumió el método abductivo, que desarrolla de manera simultánea ambas fases; por ello, la investigación proyectual es, simultáneamente, información y solución arquitectónica que, de manera permanente, va evolucionando de conjetura en conjetura experimentada. Para problematizar la investigación, se considera de manera amplia la fase de información como investigación proyectual.

La investigación proyectual es una estrategia didáctica en la formación del estudiante de arquitectura, quien no se ejercita de una forma eficaz, debido a diversos factores que influyen en la enseñanza-aprendizaje y en la manera de proyectar, lo que se ha visto reflejado en los pro- yectos arquitectónicos recurrentes, sin propuestas innovadoras, por parte de los estudiantes de las facultades de arquitectura del sistema global y especialmente latinoamericano. Dicha estrategia es un procedimiento relevante, que se viene implementando en el ámbito académico de la formación del arquitecto que se evidencia en América Latina, con Sarquis (2007); en Argentina, con el trabajo sobre investigación proyectual desarrollado en el libro Itinerarios del proyecto, de Jiménez Correa (2003); en Colombia, con el trabajo de investigación titulado Investigación y proyecto arquitectónico, y en México, con la obra de Tappan (2012), y su trabajo de investigación titulado La investigación proyectual: una propuesta que vincula docencia e investigación, entre otros, y que, a raíz de los resultados de la presente investigación, incrementan y consolidan esta estrategia proyectual.

No obstante lo anterior, existen diversas teorías, métodos y técnicas que abordan la investigación proyectual como actividad pedagógica: el proyecto arquitectónico Aprender Investigando, la investigación proyectual en arquitectura, la arquitectura proyectual y la investigación proyectual, la pedagogía del taller de enseñanza de la arquitectura, aprender a proyectar mediante el análisis de las decisiones del proyecto, las herramientas del aprendizaje de las escuelas de arquitectura, la estrategia desde la construcción de una investigación proyectual, y otros.

Para resolver la investigación proyectual como estrategia didáctica se generó un sistema planificado, programa que devino en el plan experimental, con el método del antes y el después del desarrollo de la investigación proyectual, como un conjunto de actividades graduadas que permitió a los estudiantes del décimo semestre del 2017-1 de la facultad de arquitectura de la Universidad Nacional del Centro del Perú, elevar su nivel proyectual.

En tal sentido, la investigación dio respuesta a la interrogante: ¿Cómo influye la aplicación de la investigación proyectual como estrategia didáctica en el proyecto del Taller de Diseño Arquitectónico?

La pregunta planteada, a su vez, determina como objetivo de trabajo determinar cómo influye la aplicación de la investigación proyectual como estrategia didáctica en el proyecto del Taller de Diseño Arquitectónico.

El desarrollo del plan experimental en todas sus partes fue útil para demostrar la hipótesis 
formulada: La aplicación de la investigación proyectual como estrategia didáctica influye significativamente en el proyecto del Taller de Diseño Arquitectónico.

La población-muestra estuvo conformada por la sección A 2017-1 del décimo semestre de la Facultad de Arquitectura de la Universidad Nacional del Centro del Perú, conformada por 13 estudiantes.

Entre las referencias básicas contextualizadas tenemos a Jiménez, (2003), mediante su artículo "Investigación y proyecto arquitectónico", cuyo propósito fue "precisar la interacción de procesos investigativos y práctico-reflexivos que superen las acciones instrumentales para dirimir las tensiones entre el hacer objetual y la construcción de subjetividad" (p. 13), bajo un enfoque cualitativo, con un diseño de investigación descriptivo; el instrumento fue una matriz de análisis. También se tuvo en cuenta la obra de Tappan (2012) en la investigación proyectual, una propuesta que vincula docencia e investigación, propósito gracias al cual logra introducir tres aspectos clave: 1) la naturaleza interdisciplinaria de un problema, 2) el acto creativo, o la fase de descubrimiento e innovación, y 3) el proceso de bocetaje en el proceso creativo, para después poder aplicarlos en docencia e investigación dentro de un marco contextual, metodológico y teórico, con un enfoque cualitativo, y cuyo diseño de investigación es descriptivo. Por otra parte, se tiene a Pina (2004), quien habla, en el proyecto de arquitectura, del rigor científico como instrumento poético (tesis doctoral). La Universidad Politécnica de Madrid, aporta el propósito de dar a conocer las bases esenciales del proceso lógico de proyectación, con un enfoque cualitativo-cuantitativo, cuyo diseño de investigación es descriptivo-correlacional. En su investigación proyectual en arquitectura, estudiada a través de los trabajos de grado de la maestría de diseño arquitectónico de la Facultad de Arquitectura y Urbanismo de la Universidad Central de Venezuela (tesis doctoral), Zamora (2012) se propuso orientar como método y estrategia de diseño las bases teóricas sobre la investigación proyectual, bajo el enfoque cualitativo-cuantitativo; como instrumento, utilizó una estructura cartográfica.

Las teorías básicas que sirvieron para la investigación proyectual en los diseños arquitectónicos que tienden a la innovación acorde con el desarrollo de la ciencia y la sociedad —lo que, a su vez, permitió describir, explicar, comprender, interpretar, ensayar e intentar la transformación del hábitat de la realidad- encuentran en primera instancia los aportes de la investigación proyectual como forma del conocimiento en arquitectura, planteados por Sarquis (2007) en sus dos libros de ficción epistemológica y ficción de lo real; itinerarios del proyecto, fueron de trascendental importancia, pues permitieron escrutar los prolegómenos de la investigación proyectual. Plantea Sarquis la arquitectura como conse- cuencia de una práctica social, un saber disciplinar, los integrantes del hábitat, lo existente, los ejemplos, los indicios y el análisis de los productos poiéticos.

Por su parte, Muntañola (2011) plantea como reflexiones analíticas la imaginación proyectual, la lógica proyectual y la arquitectura proyectual.

Otra referencia que refuerza esta investigación es la de Fernández (2013), quien desarrolló los conceptos sobre: el vacío de la teoría; qué investigar, cómo y para qué; cómo hacer un texto de investigación, y los productos y las perspectivas de los ecoproyectos. Como aporte de la investigación proyectual, el mismo autor plantea la existencia de un conocimiento proyectual; aborda sus temáticas desde la teoría general del hábitat y la investigación proyectual como base de una investigación científica, y dispone de una agenda de temas de investigación proyectual.

Se asumieron para la presente investigación los postulados y las bases teóricas pedagógicas del constructivismo de Ferreiro (2007, p. 27), que indicaron sus aportes del siguiente paradigma constructivista:

- La investigación proyectual como estrategia didáctica del Taller de Diseño Arquitectónico es cognoscible.

- Todo estudiante y todo docente de arquitectura son capaces de conocer la investigación proyectual como estrategia didáctica del Taller de Diseño Arquitectónico, en sucesivas aproximaciones.

- El proceso de conocimiento de la investigación proyectual como estrategia didáctica del Taller de Diseño Arquitectónico es activo, y se caracteriza por la función de la conciencia y los sentimientos del sujeto que aprende.

- Los conocimientos de la investigación proyectual como estrategia didáctica del Taller de Diseño Arquitectónico no son innatos ni están dados a priori, sino que son construidos por los estudiantes y los docentes de arquitectura. Estos se apropian de ellos mediante la actividad y el lenguaje.

- El estudiante de arquitectura que aprende no es el único responsable del proceso de construcción de su conocimiento: el ambiente es condición para su desarrollo.

Para la teoría del proyecto de arquitectura, se tuvo como base desde Vitruvio (2002) y los diez libros de arquitectura hasta los contemporáneos Le Corbusier (1959), y su Mensaje a los estudiantes de arquitectura; Alexander (1976), y su Ensayo sobre la síntesis de la forma; Quaroni (1980), con Proyectar un edificio: ocho lecciones de arquitectura; Purini (1984), con La arquitectura didáctica; Munari (1990), con Diseño y comunicación visual; Muñoz (2016), con El proyecto de arquitectura, concepto, proceso y representación, y la secuencia de aportes teóricos hasta la actualidad. Del ámbito latinoamericano, se tomaron en 
cuenta los aportes de Villagrán (1988), con su teoría de la arquitectura, y del contexto peruano, Ludeña (1997), y La arquitectura en el Perú del siglo XX.

Sobre la vigencia de los estudios y los estados del arte, existe una gama amplia de trabajos, de los cuales, en función de la variable Investigación proyectual como estrategia didáctica, se citan los siguientes artículos: de Bhooshan (2017), "Pensamiento de diseño paramétrico: un estudio de investigación arquitectónica integrada en la práctica"; de Xue y Desmet (2019), "Investigación introspectiva para la investigación de diseño basada en la experiencia", y de Khodeir (2018), "Métodos de aprendizaje combinado como un enfoque para enseñar la gestión de proyectos a estudiantes de arquitectura".

Para la variable El proyecto del Taller de Diseño Arquitectónico, se cita de AboWardah (2019): "Cerrar la brecha entre las fases de investigación y diseño esquemático en la enseñanza de proyectos de graduación arquitectónica", y de Ghonim y Eweda (2019), "Perspectivas de los instructores sobre la pedagogía de los proyectos de graduación arquitectónica: un estudio cualitativo".
El tema central de la investigación proyectual como estrategia didáctica no solo se mantiene vigente, sino que, además, sigue encontrando nuevas aplicaciones con base en el avance de las nuevas teorías o las nuevas tecnologías de informática en el sistema problemático del aprendizaje del Taller de Diseño Arquitectónico.

\section{Metodología}

El estudio cuasi experimental se llevó a cabo con la participación de estudiantes del Taller de Diseño 10, sección A, de la Facultad de Arquitectura de la Universidad Nacional del Centro del Perú, del ciclo 2017-1; del décimo semestre final de carrera. En la entrada al taller se aplicó el cuestionario Visual-Auditivo-Kinestésico (VAK), test de programación neurolingüística que determinó el tipo de estilo de aprendizaje de los estudiantes como visual en el $56 \%$; auditivo, en el $17 \%$, y kinestésico, en el $28 \%$. Una población heterogénea en sus estilos de aprendizaje y de proyectación arquitectónica que luego se tuvo en cuenta en la implementación de la metodología de investigación.

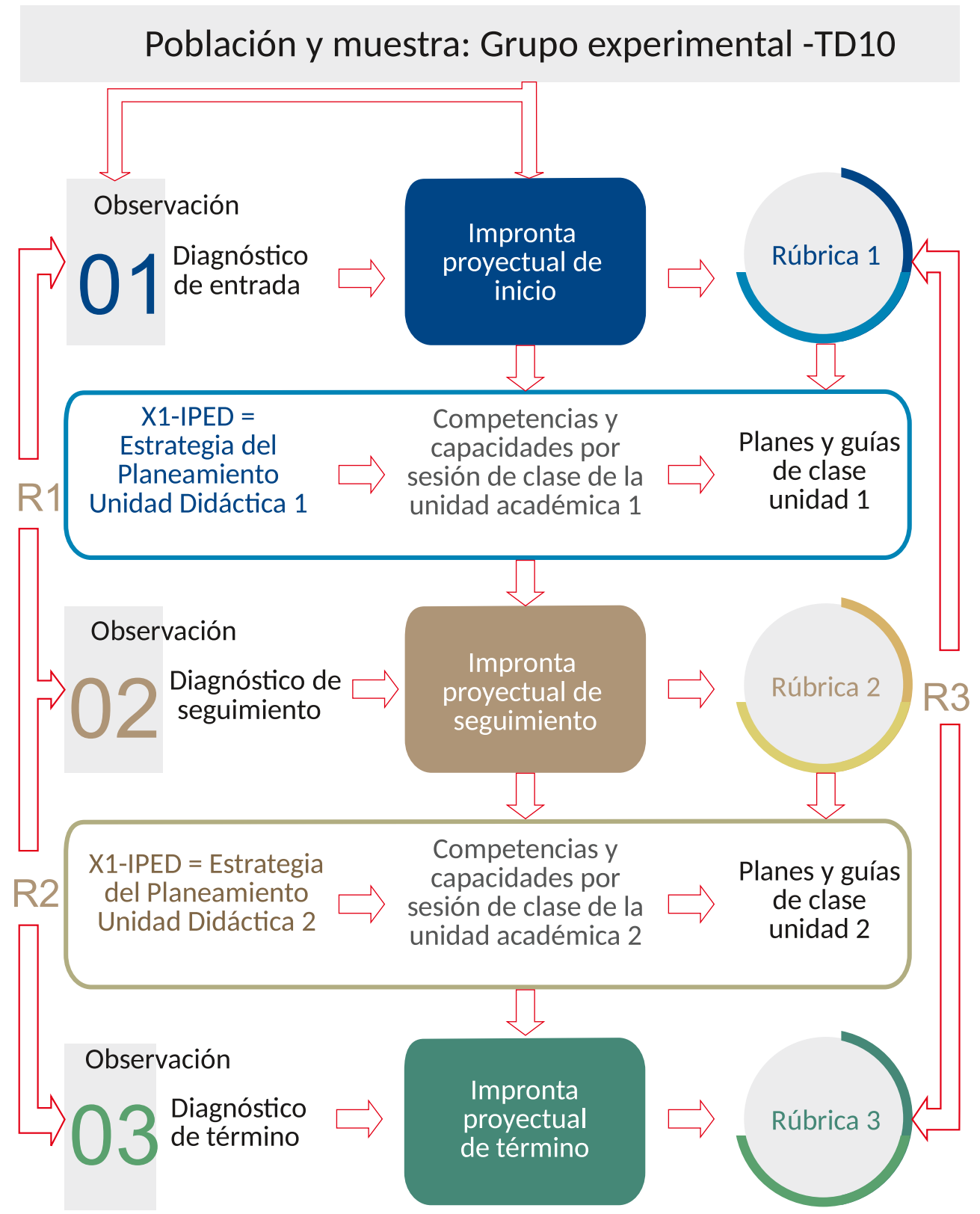

Figura 1. Estructura metodológica y procedimiento experimental, se gráfica el diseño de un solo grupo G1: O1 X O2 X O3 con sus resultados 1,2 y 3 . Fuente: Sierra (2001) y Hernández et al. (2014). 


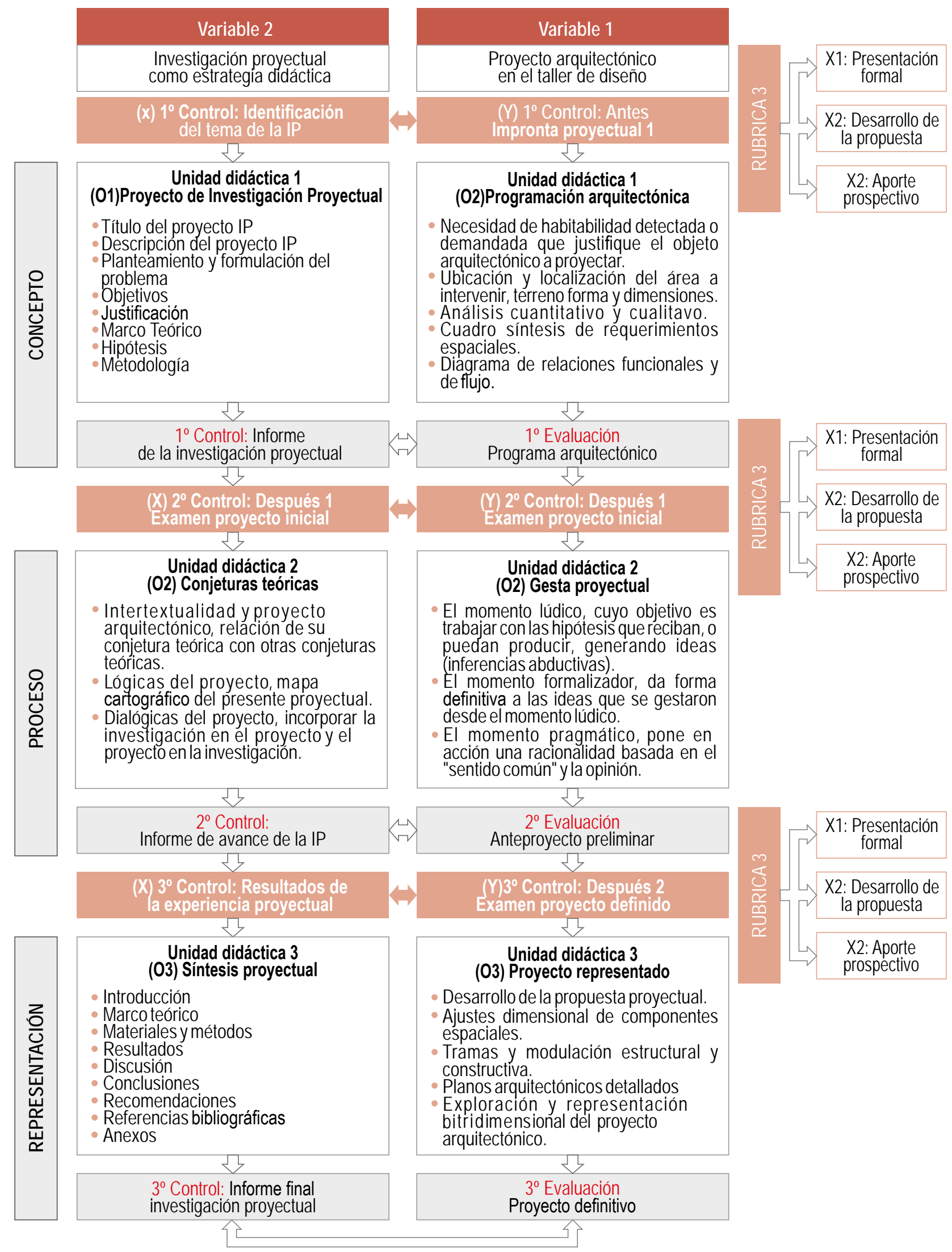

(A) Figura 2. Planeamiento, ejecución, control y evaluación del programa experimental.

Fuente: elaboración propia (2017). CC BY-NC
El tipo de investigación, dada su finalidad, se caracterizó por ser de corte aplicada; por su alcance temporal, como seccional; por su profundidad, como explicativa; por su amplitud, como microsociológica; por sus fuentes, como mixta; por su carácter, como cuantitativa, y por su naturaleza, como cuasi experimental, caracterizaciones tomadas de Sierra (2001) y de Hernández y Mendoza (2018).

En la figura 1 se observan la estructura metodológica y el procedimiento experimental de las variables independientes X1 y X2, las cuales tienen como reactivo la estrategia de planeamiento e implementación de esta en las unidades didácticas 1 y 2.
El método del planeamiento experimental con la estrategia de la calidad, productividad y competitividad se estructuró de manera sistémica con la estrategia de calidad, productividad y competitividad, propuesta por Deming.

En la figura 2 se muestra el planeamiento del programa experimental estructura en el sílabo, y su proceso de ejecución, control y evaluación experimental, que se verá reflejado en los resultados.

La evaluación del aprendizaje por competencias aplicado al programa experimental está representado en la figura 3 . Al inicio de cada una de las tres unidades académicas del taller 


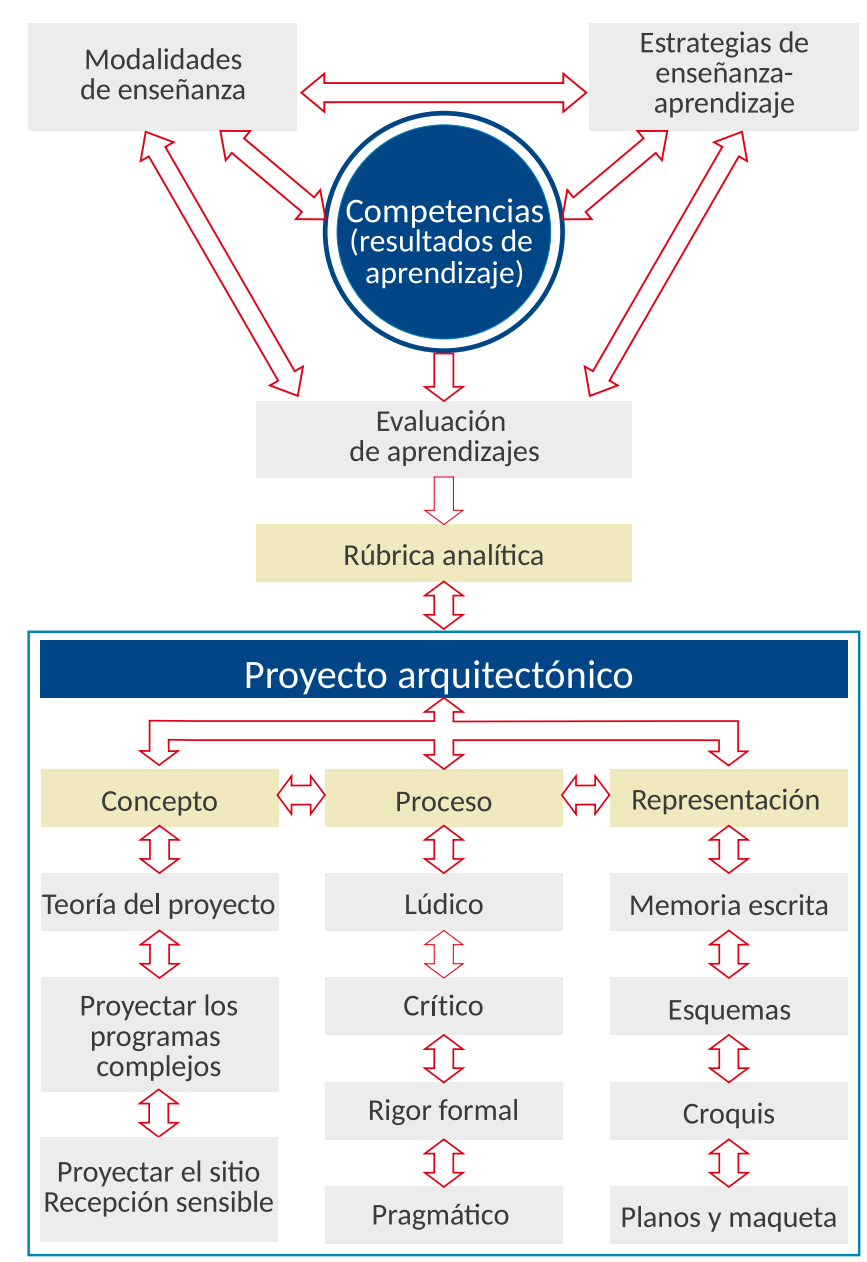

Nota: en la impronta proyectual este proceso se da de manera instantánea.

Figura 3. Evaluación del aprendizaje del proyecto arquitectónico.

Fuente: elaboración propia (2017), con base en Mezarina et al. (2016). CC BY-NC

(v) Figura 4. Conjunción de saberes.

Fuente: Sarquis (2014, p. 16).

Libertad artística

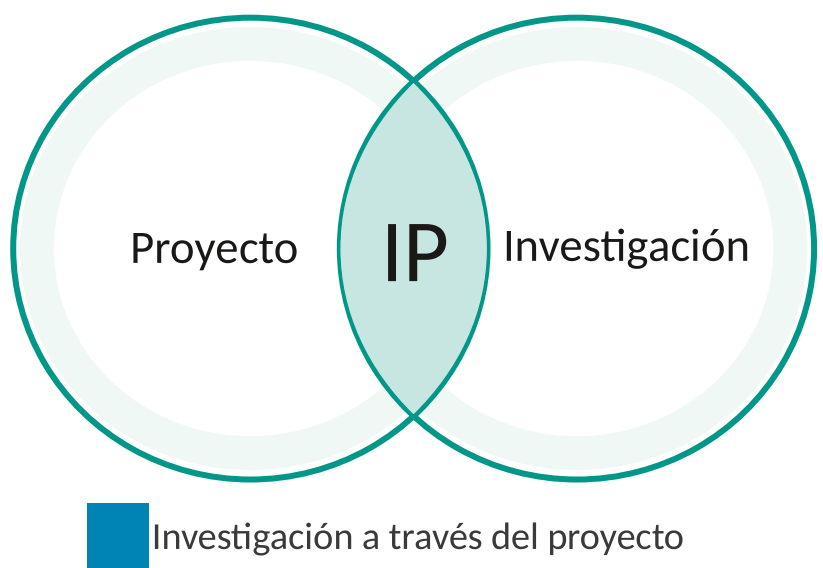

Desarrollo proyectual
Análisis de proyectos Análisis topológico

\begin{tabular}{|c||c|}
\hline \multicolumn{2}{|c|}{ Desempeños a evaluar } \\
\hline Desempeños & Indicadores \\
\hline \multirow{2}{*}{ Desempeño 1 } & Actividad \\
\cline { 2 - 2 } & Proceso \\
\cline { 2 - 2 } & Impacto \\
\hline \multirow{2}{*}{ Desempeño 2 } & Actividad \\
\cline { 2 - 2 } & Proceso \\
\hline \multirow{2}{*}{ Impacto } \\
\hline \multirow{2}{*}{ Desempeño 3 } & Actividad \\
\cline { 2 - 2 } & Proceso \\
\cline { 2 - 2 } & Impacto \\
\hline
\end{tabular}

Criterios de los indicadores

\begin{tabular}{|c|c|c|c|}
\hline \multicolumn{4}{|c|}{ Escala de valoración } \\
\hline Muy bueno (4) & Bueno (3) & Regular (2) & Deficiencia (1) \\
\hline $\begin{array}{c}\text { Jerarquía-Detallista } \\
\text { Inclusivo } \\
(100 \%-81 \%)\end{array}$ & $\begin{array}{c}\text { Jerarquía-Detallista } \\
\text { Inclusivo } \\
(80 \%-61 \%)\end{array}$ & $\begin{array}{c}\text { Jerarquía-Detallista } \\
\text { Inclusivo } \\
(60 \%-41 \%)\end{array}$ & $\begin{array}{c}\text { Jerarquía-Detallista } \\
\text { Inclusivo } \\
(40 \%-0 \%)\end{array}$ \\
\hline $\begin{array}{c}\text { Jerarquía-Detallista } \\
\text { Inclusivo } \\
(100 \%-81 \%)\end{array}$ & $\begin{array}{c}\text { Jerarquía-Detallista } \\
\text { Inclusivo } \\
(80 \%-61 \%)\end{array}$ & $\begin{array}{c}\text { Jerarquía-Detallista } \\
\text { Inclusivo } \\
(60 \%-41 \%)\end{array}$ & $\begin{array}{c}\text { Jerarquía-Detallista } \\
\text { Inclusivo } \\
(40 \%-0 \%)\end{array}$ \\
\hline $\begin{array}{c}\text { Jerarquía-Detallista } \\
\text { Inclusivo } \\
(100 \%-81 \%)\end{array}$ & $\begin{array}{c}\text { Jerarquía-Detallista } \\
\text { Inclusivo } \\
(80 \%-61 \%)\end{array}$ & $\begin{array}{c}\text { Jerarquía-Detallista } \\
\text { Inclusivo } \\
(60 \%-41 \%)\end{array}$ & $\begin{array}{c}\text { Jerarquía-Detallista } \\
\text { Inclusivo } \\
(40 \%-0 \%)\end{array}$ \\
\hline
\end{tabular}

Descriptores de nivel de desempeño de proyecto arquitectónico se aplicó la prueba de entrada como impronta proyectual, evaluada como solución arquitectónica repentina, corta y rápida. Momento de síntesis, representado en láminas de dibujo arquitectónico y maqueta física, que muestra la capacidad y aptitud del estudiante para resolver problemas arquitectónicos en una sola sesión. Se plasma una solución o una propuesta arquitectónica para un programa arquitectónico en un terreno dado, sobre la base del tema de investigación proyectual (idea generatriz) que da el énfasis a la propuesta cuya solución resuelve un problema de hábitat planteado.

En la figura 4, en la conjunción de saberes, se muestra la investigación proyectual como una intersección entre la investigación programática del proyecto arquitectónico y la solución del objeto arquitectónico. Dicho proceso se asume desde el programa general hasta la toma de decisiones dadas por la impronta proyectual.

El Instrumento rúbrica analítica es el que midió la aplicación del programa experimental, la cual, a su vez, fue construida de acuerdo con las características mostradas en la tabla 1.

La rúbrica de evaluación del proyecto de arquitectura se soportó en la investigación-acción, caracterizada por ser práctica, y que condujo a la mejora del ensayo proyectual, participativa y colaborativa, al ser aplicada como autoevaluación, coevaluación y heteroevaluación, y 
crítica, lo que permitió efectuar la retroalimentación como teoría, métodos y técnicas aplicadas a la enseñanza-aprendizaje del Taller de Diseño Arquitectónico. Así, la rúbrica de evaluación es vinculante con la evaluación de los procesos del proyecto del Taller de Diseño Arquitectónico, y fundamenta los resultados del proyecto de arquitectura en la formación del futuro arquitecto.

El proyecto arquitectónico, como producto del proceso del diseño arquitectónico, en los momentos actuales, sigue siendo evaluado de manera arbitraria e intuitiva, ya que los docentes no cuentan con instrumentos consistentes para efectuar dicho proceso. Con este instrumento se garantizan la autoevaluación, la coevaluación y la heteroevaluación.

\section{Resultados}

La experimentación práctica como profesionales arquitectos en el ejercicio de la docencia y los estudiantes en su formación profesional como arquitectos, llevada a cabo con una propuesta de aprendizaje a través de la investigación proyectual como estrategia didáctica en el proyecto del Taller de Diseño Arquitectónico, fue mutua, de docente y estudiante. Fue deconstruida en todo el proceso experimental y reconstruida en los resultados en un sistema bastante complejo, Ile- no de detalles resumidos en los resultados obtenidos y en la contrastación que la teoría y sus avances nos permiten detallar.

El tratamiento cualitativo de los datos se obtuvo de la evaluación del material gráfico que se presenta como evidencia empírica de los resultados, tal como se reseña en la figura 5.

Los resultados gráficos de la impronta proyectual diagnóstica de entrada se presentan en la figura 6 , en la que se observa, además, un gran potencial de diseño formal y con información muy elemental y básica de una formación en investigación proyectual.

Los resultados gráficos de la impronta proyectual de seguimiento se presentan en la figura 7 , en la que se observa la aplicación de la investigación proyectual en la conjetura teórica del proyecto, con manejo teórico-conceptual aplicado al tema central urbano y sus proyectos arquitectónicos individuales.

Los resultados gráficos de la impronta proyectual de término se presentan en la figura 8 , en la que se observa la aplicación de la investigación proyectual interactuando con el proyecto urbano y sus proyectos arquitectónicos individuales, reflejados en su partido arquitectónico y su anteproyecto preliminar. $\rightarrow$ Figura 5.

Implementación del

planeamiento, la ejecución control y la evaluación

Fuente: elaboración propia (2017). CC BY-NC

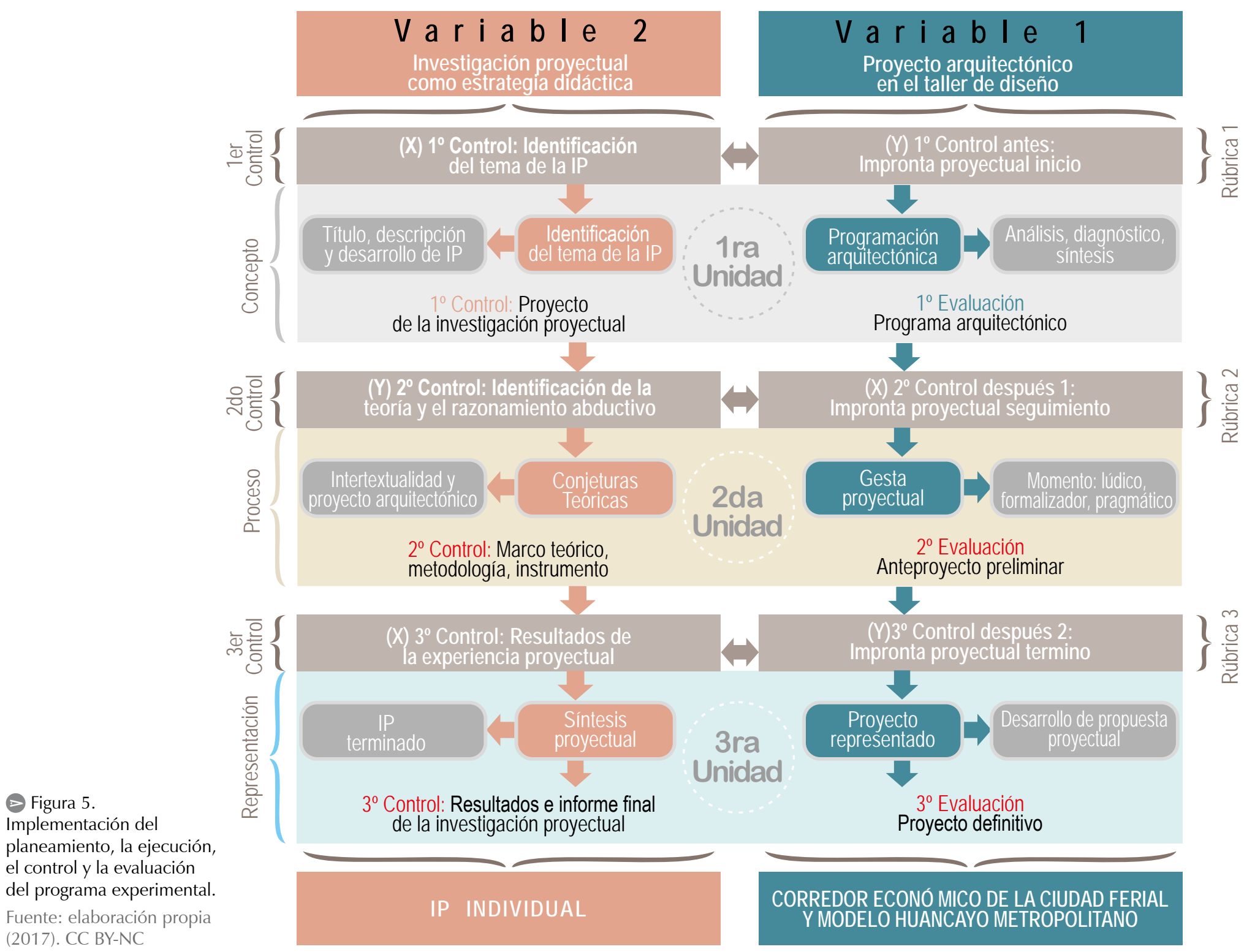



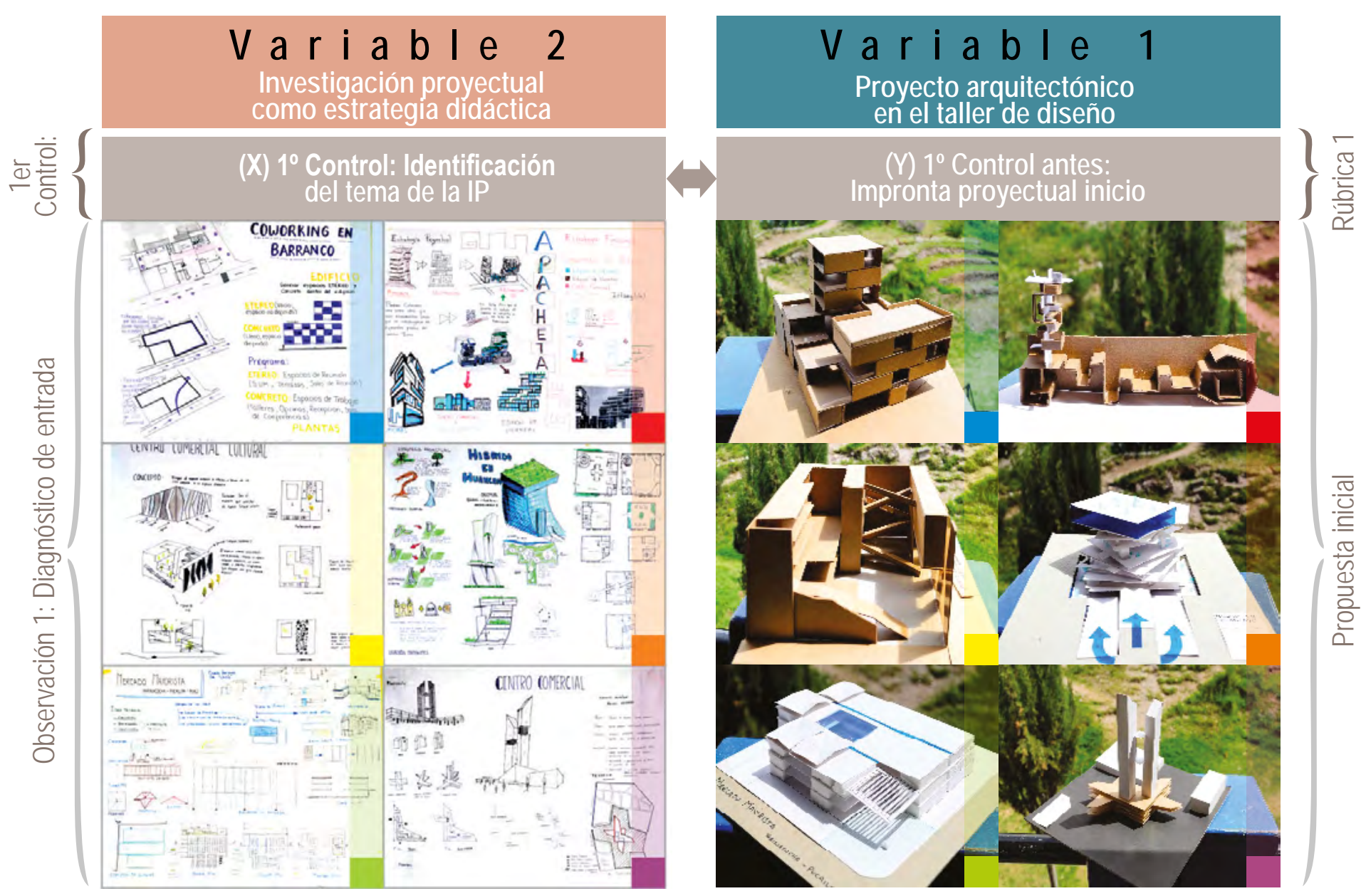

(A) Figura 6. Impronta proyectual diagnóstica de entrada. Fuente: elaboración propia (2017). CC BY-NC
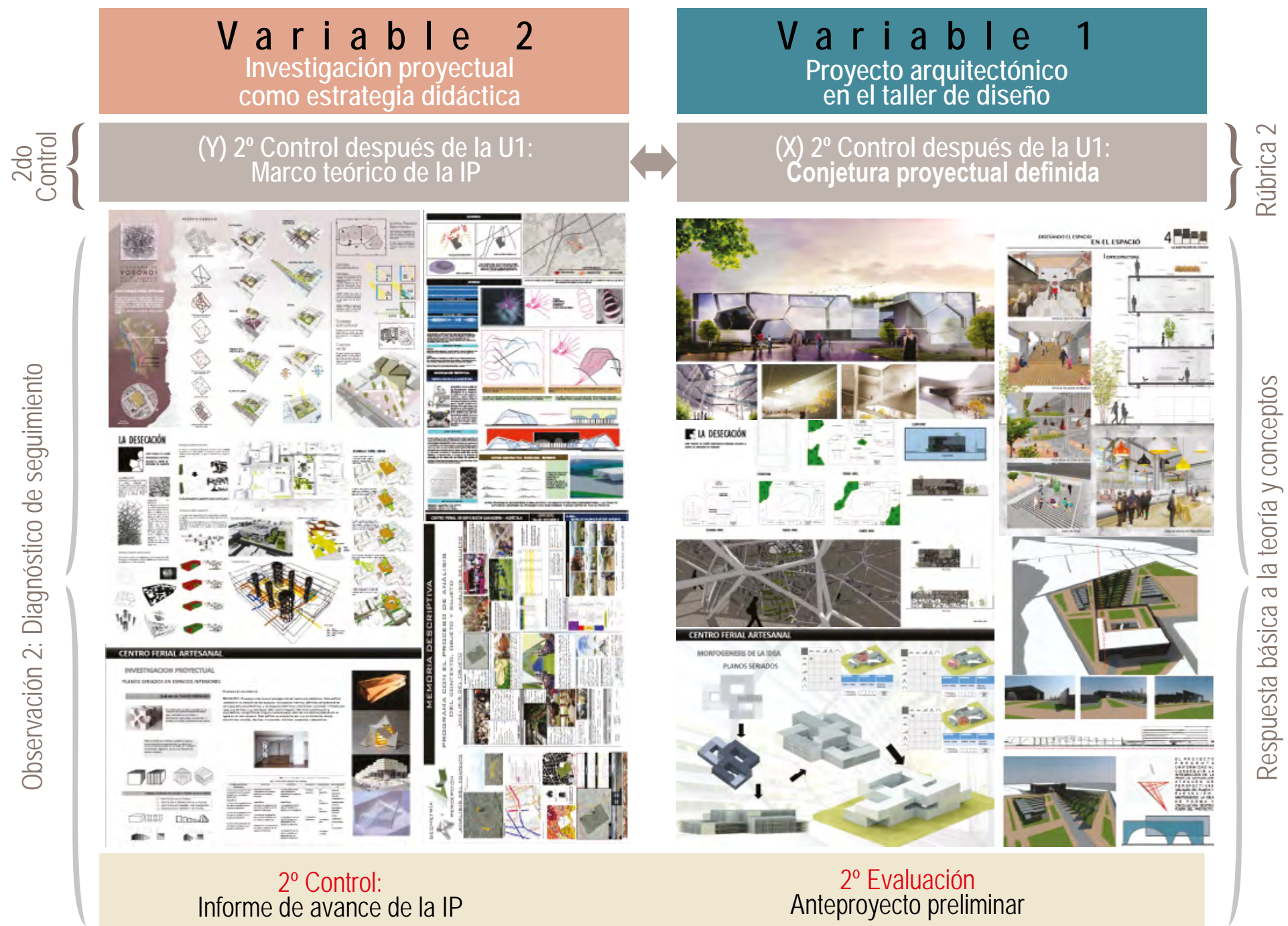

Informe de avance de la IP

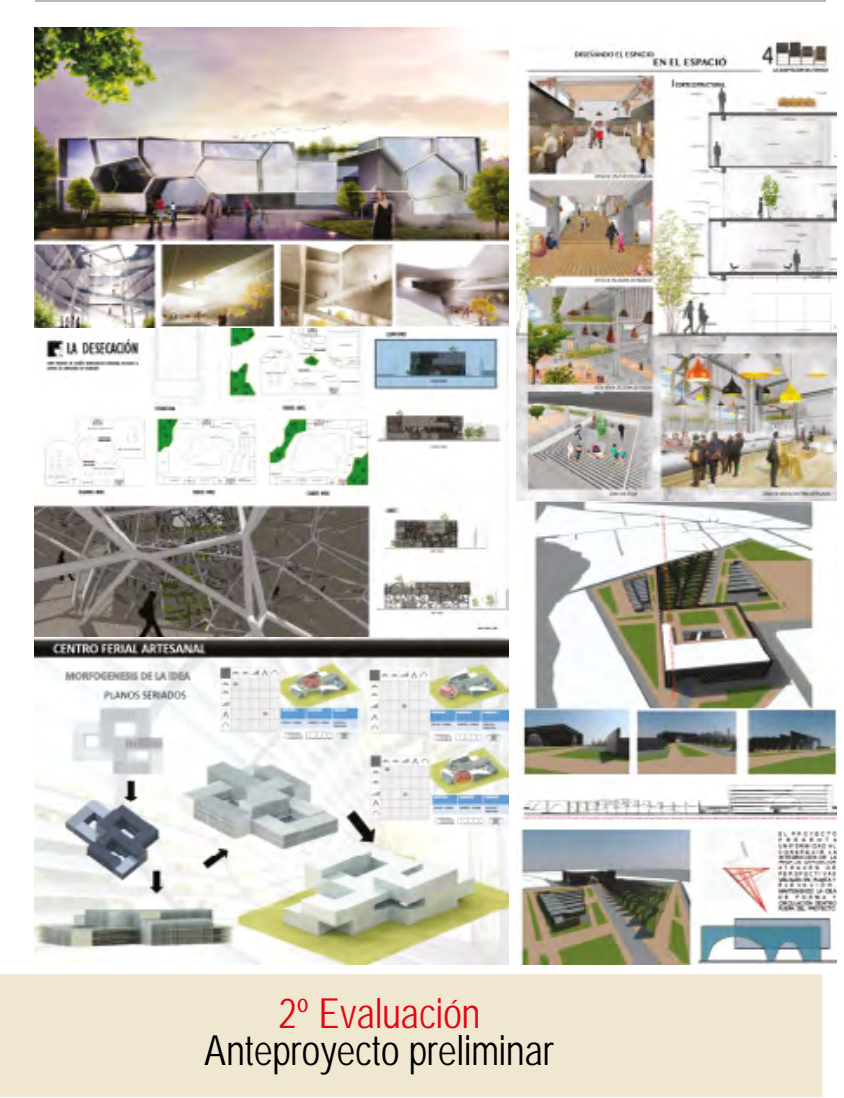

(A) Figura 7. Impronta proyectual de seguimiento.

Fuente: elaboración propia (2017). CC BY-NC 

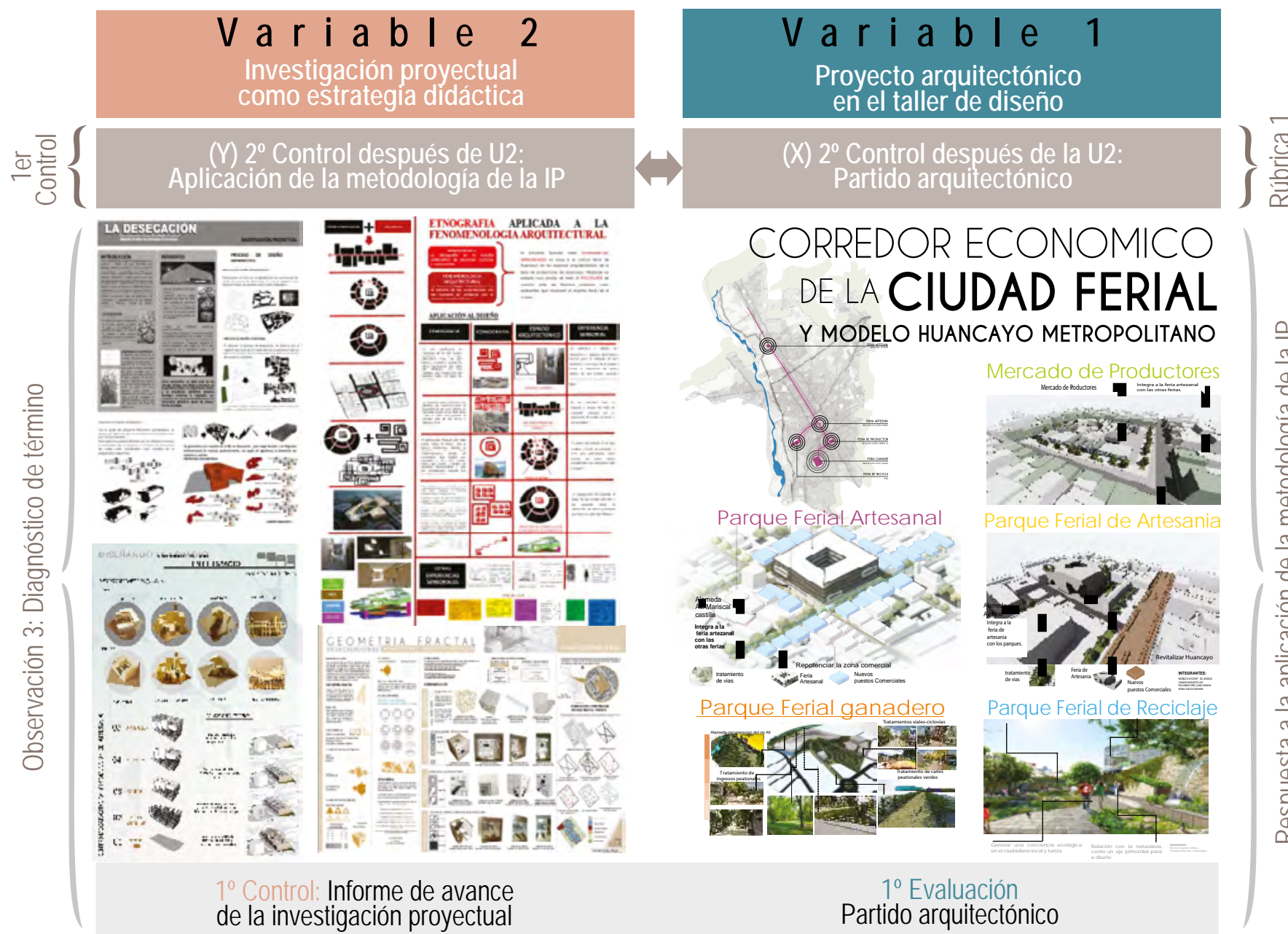
DE LA CIUDAD FERIAL ID Y MODELO HUANCAYO METROPOLITANO
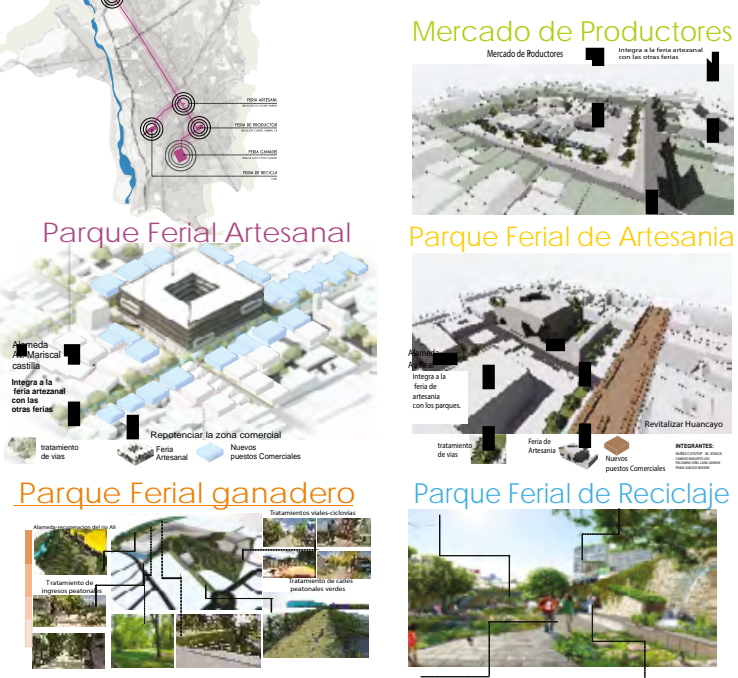

10 Evaluación de la investigación proyectual Partido arquitectónico

(4) Figura 8. Impronta proyectual de término. Fuente: elaboración propia (2017). CC BY-NC
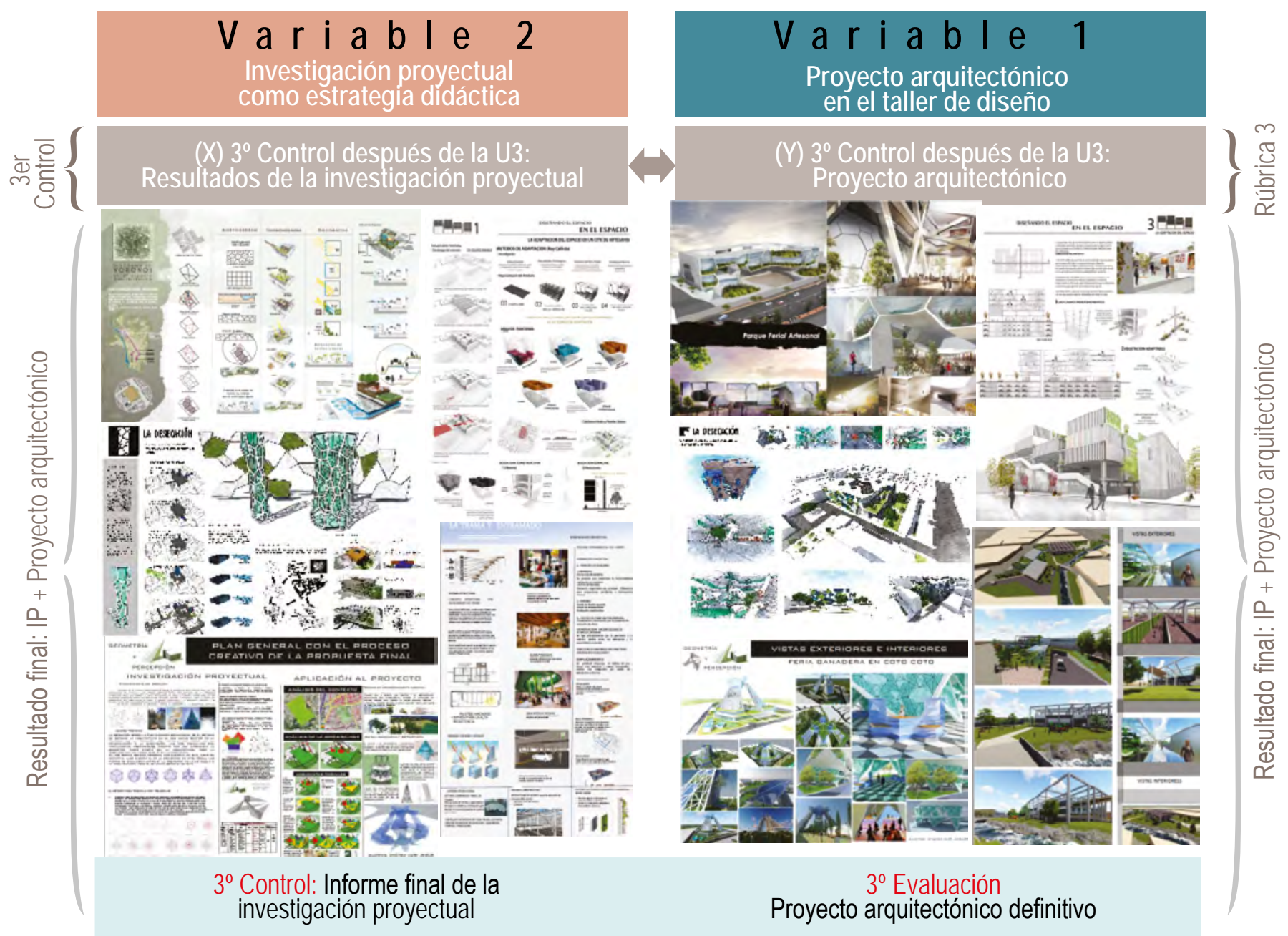

(A) Figura 9. Proyecto arquitectónico final. 


\begin{tabular}{cccc} 
& \multicolumn{3}{c}{ Kolmogorov-Smirnov } \\
& Estadístico & gl & Sig. \\
\hline $\begin{array}{c}\text { Impronta } \\
\text { proyectual 1 }\end{array}$ & 0,192 & 13 & $0,200^{*}$ \\
$\begin{array}{c}\text { Impronta } \\
\text { proyectual 2 }\end{array}$ & 0,225 & 13 & 0,072 \\
$\begin{array}{c}\text { Impronta } \\
\text { proyectual 3 }\end{array}$ & 0,308 & 13 & 0,001 \\
\hline
\end{tabular}

(A) Tabla 2. Prueba de normalidad general.

Fuente: elaboración propia (2017). CC BY-NC

Los resultados gráficos del proyecto arquitectónico final se presentan en la figura 9, en la que se observan los resultados finales de la investigación proyectual interactuando con el proyecto urbano y sus proyectos arquitectónicos individuales, reflejados en su proyecto arquitectónico final.

Como correlato de la evidencia gráfica empírica, se desarrollan los resultados cuantitativos de las rúbricas de evaluación.

El tratamiento cuantitativo de los datos se hizo mediante la estadística descriptiva e inferencial, procesados los datos mediante el Spss 24.

Al inicio se procedió con la prueba de normalidad general con base en la regla teórica para decisión estadística:

Regla teórica:

- Si el valor de significancia es >0,05 es normal.

- Si el valor de significancia es < 0,05 no es normal.

De la tabla 2 se obtienen los siguientes datos de distribución normal:

- Impronta proyectual 1: 0,200>0,05 es normal.

- Impronta proyectual 2: 0,072>0,05 es normal.

- Impronta proyectual 3: 0,001<0,05 no es normal.

Decisión estadística: por lo anterior, al no presentar normalidad en la totalidad de evaluaciones, se aplicó la prueba no paramétrica de Wilcoxon.

Una vez ejecutada la prueba de normalidad para la contratación de las hipótesis específicas se concluyó que al haber uno o más datos de no normalidad, se debía utilizar la prueba no paramétrica de Wilcoxon.

Con los resultados preliminares, y habiendo establecido como el estadístico de prueba la prueba no paramétrica de Wilcoxon, se procedió a la prueba de la hipótesis general.

Regla teórica:

- $\mathrm{H}_{\mathrm{O}}$ : No existe diferencia significativa entre la evaluación inicial i y la evaluación inicial j.

- $\mathrm{H}_{1}$ : Si existe diferencia significativa entre la evaluación inicial i y la evaluación inicial j.

\begin{tabular}{cccc} 
& \multicolumn{4}{c}{ Estadísticos de prueba } \\
& $\begin{array}{c}\text { Improntas } \\
\text { proyectuales: } \\
\mathbf{1 - 2}\end{array}$ & $\begin{array}{c}\text { Improntas } \\
\text { proyectuales: } \\
\mathbf{1 - 3}\end{array}$ & $\begin{array}{c}\text { Improntas } \\
\text { proyectuales: } \\
\mathbf{2 - 3}\end{array}$ \\
\hline Z &,$- 861 \mathrm{~b}$ & $-2,439 \mathrm{c}$ & $-3,071 \mathrm{c}$ \\
\hline $\begin{array}{c}\text { Sig. } \\
\text { asintótica } \\
\text { (bilateral) }\end{array}$ & 0,389 & 0,015 & 0,002 \\
\hline
\end{tabular}

(A) Tabla 3. Estadísticos de prueba de Wilcoxon general. Fuente: elaboración propia (2017). CC BY-NC

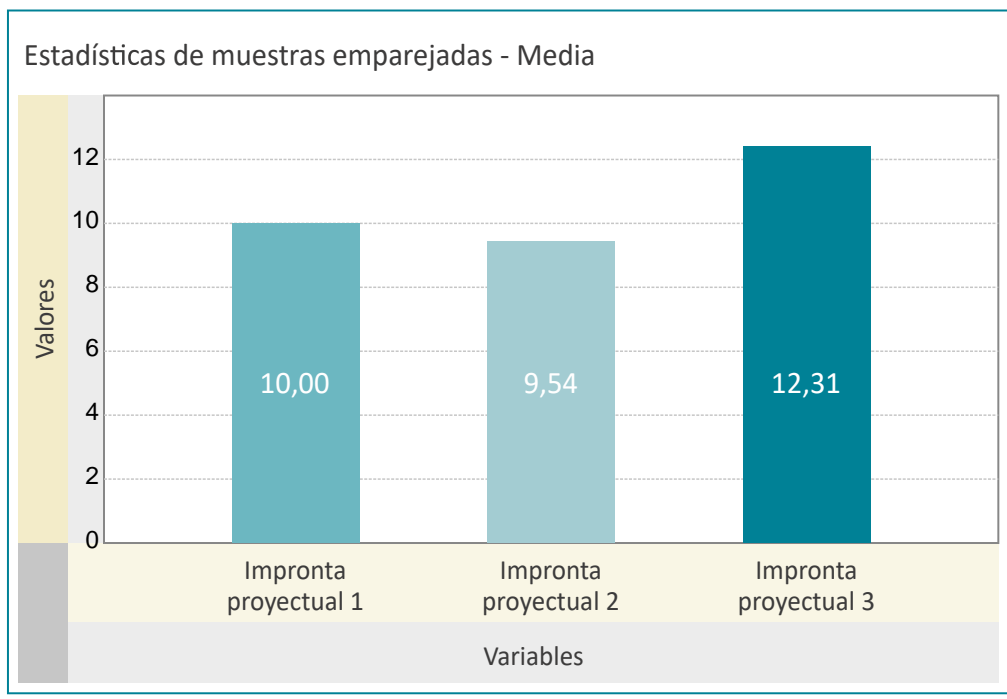

(A) Figura 10. Medias de la variable impronta proyectual. Fuente: elaboración propia (2017). CC BY-NC

\begin{tabular}{|c|c|c|c|c|}
\hline \multicolumn{4}{|c|}{ Estadísticos de prueba } & \multirow{3}{*}{$\begin{array}{l}\text { Tabla 4. Estadísticos } \\
\text { de prueba de Wilcoxon } \\
\text { de la dimensión } \\
\text { presentación formal. }\end{array}$} \\
\hline & $\begin{array}{l}\text { Presentación } \\
\text { formal: } 1-2\end{array}$ & $\begin{array}{c}\text { Presentación } \\
\text { formal: 1-3 }\end{array}$ & $\begin{array}{c}\text { Presentación } \\
\text { formal: } 2-3\end{array}$ & \\
\hline Z & $-1,011$ & $-2,453$ & $-2,781$ & \\
\hline $\begin{array}{c}\text { Sig. } \\
\text { asintótica } \\
\text { (bilateral) }\end{array}$ & 0,312 & 0,014 & 0,005 & $\begin{array}{l}\text { presentación formal. } \\
\text { Fuente: elaboración } \\
\text { propia (2017). CC } \\
\text { BY-NC }\end{array}$ \\
\hline
\end{tabular}

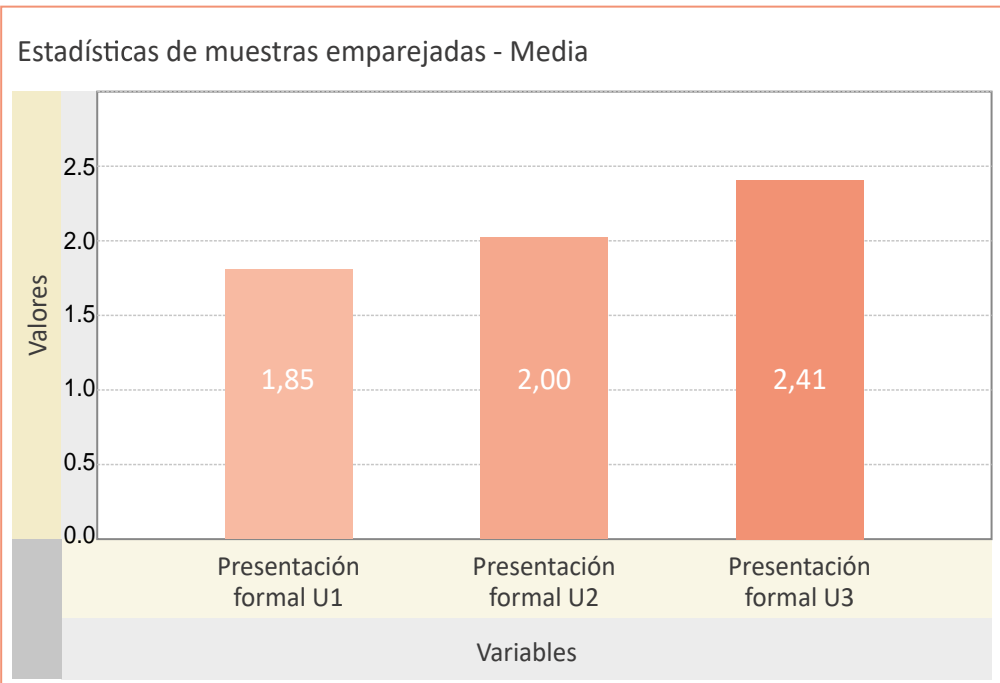

A Figura 11. Medias de la dimensión presentación formal. Fuente: elaboración propia (2017). CC BY-NC 
Tabla 5. Estadísticos de prueba de Wilcoxon de la dimensión desarrollo de la propuesta.

Fuente: elaboración propia (2017). CC BY-NC

\begin{tabular}{|c|c|c|c|}
\hline \multicolumn{4}{|c|}{ Estadísticos de prueba } \\
\hline & $\begin{array}{c}\text { Aporte } \\
\text { prospectivo: } \\
1-2\end{array}$ & $\begin{array}{c}\text { Aporte } \\
\text { prospectivo: } \\
1-3\end{array}$ & $\begin{array}{c}\text { Aporte } \\
\text { prospectivo: } \\
2-3\end{array}$ \\
\hline Z & $-1,059 b$ & $-1,809 c$ & $-2,739 c$ \\
\hline $\begin{array}{c}\text { Sig. } \\
\text { asintótica } \\
\text { (bilateral) }\end{array}$ & 0,289 & 0,070 & 0,006 \\
\hline
\end{tabular}

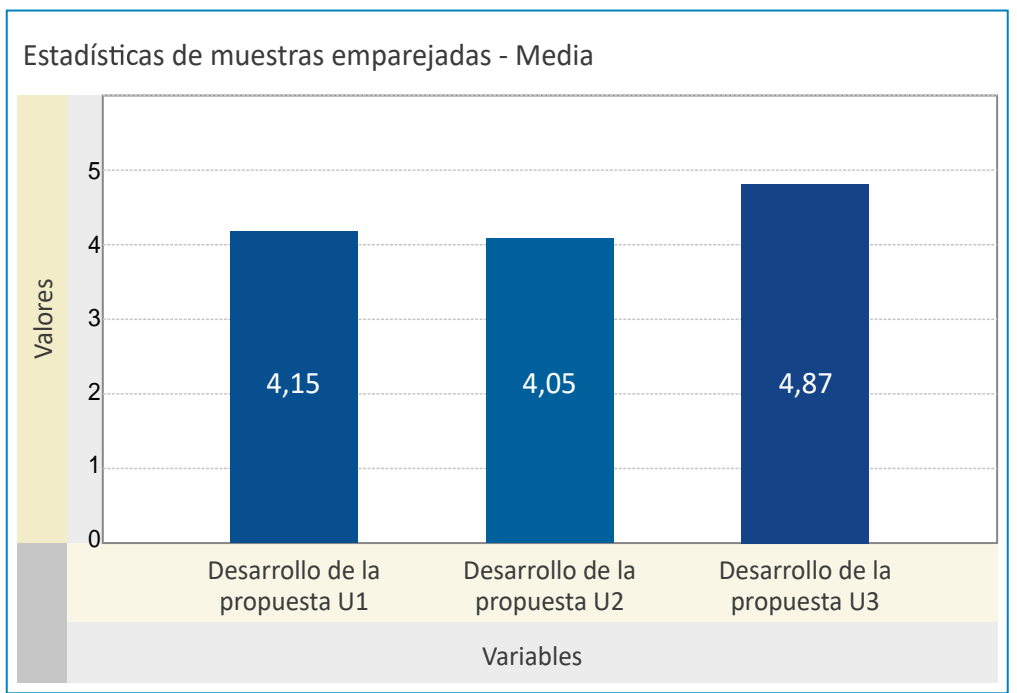

(A) Figura 12. Medias de la dimensión desarrollo de la propuesta. Fuente: elaboración propia (2017). CC BY-NCEm auc te ta

$\rightarrow$ Tabla 6. Estadísticos de prueba de Wilcoxon de la dimensión aporte prospectivo. Fuente: elaboración propia (2017). CC BY-NC

\begin{tabular}{|c|c|c|c|}
\hline \multicolumn{4}{|c|}{ Estadísticos de prueba } \\
\hline & $\begin{array}{c}\text { Desarrollo } \\
\text { de la } \\
\text { propuesta: } \\
1-2\end{array}$ & $\begin{array}{c}\text { Desarrollo } \\
\text { de la } \\
\text { propuesta: } \\
1-3\end{array}$ & $\begin{array}{c}\text { Desarrollo } \\
\text { de la } \\
\text { propuesta: } \\
2-3\end{array}$ \\
\hline Z &,$- 451 b$ & $-1,875 c$ & $-2,607 c$ \\
\hline $\begin{array}{c}\text { Sig. } \\
\text { asintótica } \\
\text { (bilateral) }\end{array}$ & 0,652 & 0,061 & 0,009 \\
\hline
\end{tabular}

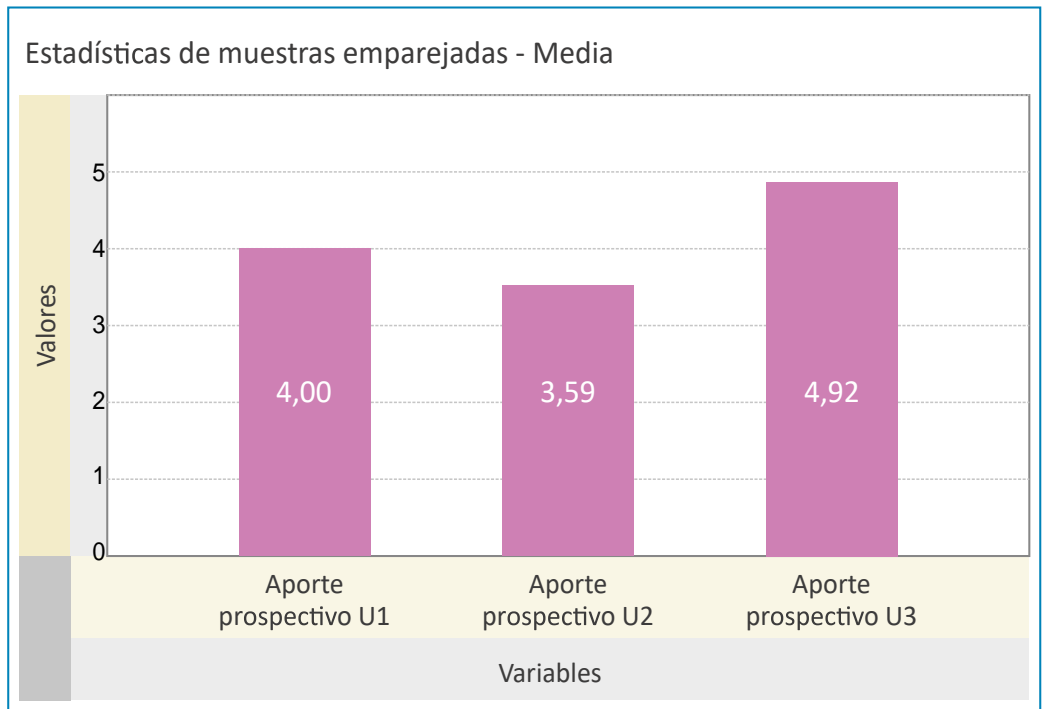

A Figura 13. Medias de la dimensión aporte prospectivo. Fuente: elaboración propia (2017). CC BY-NC
La prueba de Wilcoxon demuestra que en el primer caso no se rechaza la $\mathrm{H}_{\mathrm{O}}(p 1=0,389>$ $0,05)$, y en el segundo y el tercero sí se rechaza la $\mathrm{H}_{\mathrm{O}}(p 2=0,015<0,05 ; p 3=0,002<0,05)$.

Decisión estadística: de la tabla 3 y la figura 10 se deduce que los resultados de la impronta proyectual 3 son los que muestran un mayor promedio, lo cual indica que la aplicación de la investigación proyectual ha influido significativamente en los procedimientos y el proyecto arquitectónico desarrollado en el Taller de Diseño Arquitectónico.

Prueba de hipótesis específicas de la dimensión presentación formal.

Regla teórica:

- $\mathrm{H}_{\mathrm{O}}$ : No existe diferencia significativa entre los resultados de la presentación formal de la unidad i y los resultados de la presentación formal de la unidad $j$.

- $\mathrm{H}_{1}$ : Si existe diferencia significativa entre los resultados de la presentación formal de la unidad i y los resultados de la presentación formal de la unidad $\mathrm{j}$.

La prueba de Wilcoxon demuestra que en el primer caso no se rechaza la $\mathrm{H}_{\mathrm{O}}(p 1=0,312>$ $0,05)$ y en el segundo y el tercero sí se rechaza la $\mathrm{H}_{\mathrm{O}}(p 2=0,014<0,05 ; p 3=0,005<0,05)$.

Decisión estadística: de la Tabla 4 y la Figura 11 se deduce que la dimensión presentación formal de la impronta proyectual de la unidad 3 es la que muestra un mayor promedio, lo cual, a su vez, indica que la aplicación de la investigación proyectual ha influido significativamente en los procedimientos y el proyecto arquitectónico desarrollado en el Taller de Diseño Arquitectónico.

Hipótesis específica de la dimensión desarroIlo de la propuesta.

Regla teórica:

- $\mathrm{H}_{\mathrm{O}}$ : No existe diferencia significativa entre los resultados del desarrollo de la propuesta de la unidad i y del desarrollo de la propuesta de la unidad $\mathrm{j}$.

- $\mathrm{H}_{1}$ : Sí existe diferencia significativa entre los resultados del desarrollo de la propuesta de la unidad i y del desarrollo de la propuesta de la unidad $j$.

La prueba de Wilcoxon demuestra que ni en el primer ni en el segundo caso se rechazan las $\mathrm{H}_{\mathrm{O}}(p 1=0,652>0,05 ; p 2=0,061>0,05)$, y el tercero sí se rechaza la $\mathrm{H}_{\mathrm{o}} ; \mathrm{p} 3=0,009<$ 0,05).

Decisión estadística: de la tabla 5 y la figura 12 se deduce que la dimensión desarrollo de la propuesta de la impronta proyectual de la unidad 3 es la que muestra un mayor promedio, lo cual, a su vez, indica que la aplicación de la investigación proyectual ha influido significativamente en los procedimientos y el proyecto 
arquitectónico desarrollado en el Taller de Diseño Arquitectónico.

Hipótesis específica de la dimensión aporte prospectivo.

\section{Regla teórica:}

- $\mathrm{H}_{\mathrm{o}}$ : No existe diferencia significativa entre los resultados del aporte prospectivo i y los resultados del aporte prospectivo j.

- $\mathrm{H}_{1}$ : Sí existe diferencia significativa entre los resultados del aporte prospectivo i y los resultados del aporte prospectivo $\mathrm{j}$.

La prueba de Wilcoxon demuestra que ni en el primer ni en el segundo caso se rechazan las $\mathrm{H}_{\mathrm{O}}(p 1=0,289>0,05 ; p 2=0,070>0,05)$, y en el tercero si se rechaza la $\mathrm{H}_{\mathrm{o}} ; p 3=0,006$ $<0,05)$.

Decisión estadística: de la tabla 6 y figura 13 se deduce que los resultados del aporte prospectivo de la unidad 3 es la que muestra un mayor promedio, lo cual indica que la aplicación de la investigación proyectual ha influido significativamente en los procedimientos y el proyecto arquitectónico desarrollado en el Taller de Diseño Arquitectónico.

\section{Discusión}

El proyecto de taller de diseño y el proceso de enseñanza aprendizaje ha corrido en paralelo con el desarrollo de la investigación, donde en cada parte del proceso fue necesario revisar los postulados teóricos y los antecedentes en los que se basa la investigación, resolviendo situaciones concretas a través de la retroalimentación permanente que generó una dinámica interactiva de aprendizaje mutuo del docente y el estudiante. Se contrastan los resultados con la teoría y los antecedentes que lo sostienen de lo sistematizado en la experiencia de la investigación proyectual, como estrategia didáctica.

Los aportes de investigación proyectual planteados por Sarquis (2007) en sus libros Ficción epistemológica y Ficción de lo real, itinerarios del proyecto, resultado de su tesis doctoral, y que sirvieron de base para su aplicación en la maestría y el doctorado del mismo nombre en la Universidad de Buenos Aires (UBA), para nuestro caso se introdujeron como experiencia en pregrado, y hacerlo fue todo un reto. Los resultados obtenidos en la investigación proyectual como estrategia didáctica en el proyecto del Taller de Diseño Arquitectónico, han sido significativos, e indican que es una estrategia válida para su aplicación en pregrado.

Cuando en su libro El proyecto de arquitectónico aprender investigando, Jiménez (2006) demuestra la importancia que el proyecto genera conocimiento en el estudiante, lo hizo desde la óptica del juicio de expertos, a diferencia de la presente investigación, en la cual se demuestra por la evaluación de la producción de los proyectos arquitectónicos trabajados por los estudiantes, y se valida así la misma hipótesis desde un trabajo experimental.

Es factible implementar teorías y propuestas de organización en el programa experimental insertadas en el sílabo del TD10, como es el caso de la teoría de calidad, productividad y competividad, de Deming (1989), en sus cuatro procesos adaptados al programa desarrollado de planeamiento, ejecución, control y evaluación, desde la óptica y las estrategias didácticas.

Para operar la variable Proyecto del Taller de Diseño Arquitectónico, se la procesó con el marco teórico de Muñoz (2016) y su libro El proyecto de arquitectura. Desarrolla su temática en dos ámbitos: 1) el del ejercicio profesional y 2) el de la formación del arquitecto. Es el segundo aspecto el que se toma como referencia básica y se corrobora para la elaboración del instrumento rúbrica analítica, que evalúa la impronta proyectual del inicio, el seguimiento y el término de la investigación. Sus definiciones y sus conceptos sobre los detalles del proyecto arquitectónico cobran vigencia en el instrumento rúbrica analítica de evaluación y en los resultados obtenidos.

Para el desarrollo del trabajo fue necesario tomar todos los referentes concurrentes al objeto de la investigación; por ello, el trabajo de Martínez (2013) El proyecto arquitectónico como un problema de investigación tuvo como objetivo principal plantear la identificación de procesos metodológicos para desarrollar la práctica del diseño arquitectónico, que se resume en la programación arquitectónica. Su aporte fue sustancial en la confección de los ítems de la dimensión presentación formal de la rúbrica analítica de evaluación.

La aplicación de la investigación proyectual como estrategia didáctica ha influido significativamente en los procedimientos y el proyecto arquitectónico desarrollado en el Taller de Diseño Arquitectónico, que puede ser replicado para la formación del arquitecto en las diversas modalidades de talleres arquitectónicos que se dan en América Latina.

\section{Conclusiones}

Detallando la aplicación de la impronta proyectual evaluada por la rúbrica analítica en sus resultados generales y en sus tres dimensiones (presentación formal, desarrollo de la propuesta y aporte prospectivo), se tienen las siguientes conclusiones:

De la aplicación de las tres improntas proyectuales, al inicio de cada unidad y del análisis estadístico de la primera y la segunda, la primera y la tercera; la segunda y la tercera, se obtuvieron los resultados planteados en el 
proyecto de investigación que indican que la aplicación de la investigación proyectual como estrategia didáctica en la primera unidad no logra superar sus saberes previos. Y para la segunda y tercera unidades se ha superado la etapa de adecuación teniendo en el resultado final, que ha influido significativamente en los procedimientos y el proyecto arquitectónico desarrollado en el Taller de Diseño Arquitectónico.

En las dimensiones específicas, a semejanza del análisis general, se tiene para la dimensión presentación formal que los resultados de la impronta proyectual al inicio de cada unidad y del análisis estadístico de la primera y la segunda; la primera y la tercera; la segunda y la tercera, demuestran que en la primera unidad no se logra superar sus saberes previos en la dimensión presentación formal, y para la segunda y la tercera unidades se ha superado la etapa de adecuación en la aplicación de la investigación proyectual, como estrategia didáctica.
En la dimensión desarrollo de la propuesta se compararon los resultados de la impronta proyectual al inicio de cada unidad, y el análisis estadístico demuestra que en la primera y en la segunda unidad no se logra superar sus saberes previos en la dimensión desarrollo de la propuesta, y para la tercera unidad se ha superado la etapa de adecuación en la aplicación de la investigación proyectual como estrategia didáctica.

En la dimensión aporte prospectivo se compararon los resultados de la impronta proyectual al inicio de cada unidad, y el análisis estadístico de la primera y la segunda, la primera y la tercera; la segunda y la tercera demuestra que la aplicación de la investigación proyectual como estrategia didáctica en la primera y la segunda unidades no logra superar sus saberes previos en la dimensión aporte prospectivo, y para la tercera unidad se ha superado la etapa de adecuación en la aplicación de la investigación proyectual como estrategia didáctica.

\section{Referencias}

AboWardah, E. S. (2019). Bridging the gap between research and schematic design phases in teaching architectural graduation projects. Frontiers of Architectural Research, 9(1), 82-105.

https://doi.org/10.1016/j.foar.2019.04.005

Alexander, C. (1976). Ensayo sobre la síntesis de la forma (4a). Ediciones Infinito.

Bhooshan, S. (2017). Parametric design thinking: A case-study of practice-embedded architectural research. Design Studies, 52, 115-143. https://doi.org/10.1016/j.destud.2017.05.003

Deming, W. (1989). Calidad, productividad y competividad (versión es). Ed. Díaz de Santos.

Fernández, R. (2013). Inteligencia proyectual, un manual de investigación en arquitectura. Editorial Teseo.

Ferreiro, R. (2007). Estrategias didácticas del aprendizaje cooperativo, el constructivismo social: una nueva forma de enseñar y aprender. Ed. Trillas.

Ghonim, M. y Eweda, N. (2019). Instructor perspectives on the pedagogy of architectural graduation projects: A qualitative study. Frontiers of Architectural Research 8(3) 415-427. https://doi.org/10.1016/j.foar.2019.01.007

Hernández, R., Fernández, C. y Baptista, M. del P. (2014). Metodología de la invetigación (6a). McGraw-Hill/Interamericana Editores, S. A.

Hernández, R. y Mendoza, P. (2018). Metodología de la investigación. McGraw-Hill Education. liménez Correa, S. (2003). Investigación y proyecto arquitectónico. Revista Guillermo de http://bibliotecadigital.usb.edu.co/ bitstream/10819/5231/1/435-981-1-PB.pdf

Jiménez, S. (2006). El proyecto arquitectónico, aprender investigando. Universidad de San Buenaventura Cali.

Khodeir, L. M. (2018). Blended learning methods as an approach to teaching project management to architecture students. Alexandria Engineering Journal 57(4), 3899-3905. https://doi.org/10.1016/j.aej.2018.10.004

Le Corbusier, J. (1959). Mensaje a los estudiantes de arquitectura. En Biblioteca de arquitectura (Décima, Vol. 6). Ediciones Infinito.

Ludeña, W. (1997). Ideas y arquitectura en el Perú del siglo XX. Servicios Editoriales Múltiples S. A.

Martínez, P. (2013). El proyecto arquitectónico como un problema de investigación. Universidad Católica de Colombia.

Mezarina, C., Moya, N., Portillo, O., Cárdenas J. y Suasnabar, L. (2016). Modelo educativo - UNCP. Universidad Nacional del Centro del Perú.

Munari, B. (1990). Diseño y comuncación visual (10a). Gustavo Gili, S.A.

Muñoz, A. (2016). El proyecto de arquitectura, concepto proceso y representacion. Editorial Reverté.

Muntañola, J. (2011). La investigación proyectual a examen: un gran desafío a la arquitectura del siglo XXI. Conferencia inaugural de la trienal de investigación FAU-UCV 2011. Facultad de Arquitectura y Urbanismo Universidad Central de Venezuela.

http://trienal.fau.ucv.ve/documentos/ Ensayo_de_postgrado_8.pdf

Pina, R. (2004). El proyecto de arquitectura, el rigor científico como instrumento poético. Universidad Politécnica de Madrid.

Purini, F. (1984). La arquitectura didáctica. Colección de Arquitectura.

Quaroni, L. (1980). Proyectar un edificio ocho lecciones de arquitectura. Xarait Libros S. A

Sarquis, J. (2007). 1 Ficción epistemológica itinerarios del proyecto la investigacion proyectual como forma de conocimiento en arquitectura. Bibliográfika de Voros S. A.

Sarquis, J. (2014). Experiencias pedagógicas creativas. Bibliográfika de Voros S. A.

Sierra, R. (2001). Técnicas de investigación social. Universidad Panamericana.

Tappan, M. (2012). La investigación proyectual: Una propuesta que vincula docencia e investigación. En La investigación en diseño, una visión desde los posgrados en México (pp. 219-243). Universidad Autónoma de Ciudad Juárez.

Villagrán, J. (1988). Teoría de la arquitectura. Litográfica Rendón, S. A.

Vitruvio, M. (2002). Los diez libros de arquitectura (3a edición). Alianza Editorial.

Xue, H. y Desmet, P. M. A. (2019). Researcher introspection for experience-driven design research. Design Studies, 63, 37-64. https:// doi.org/10.1016/j.destud.2019.03.001

Zamora, H. (2012). La investigación proyectual en arquitectura. Universidad Central de Venezuela.

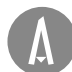



(1) Portada: Las bovedadas de Bramante.

Fotografía: Luis Alberto Martínez Camacho (2021) CC BY-NC

\section{(ब) (1) (\$)}

(A) Orientación editorial

\section{Enfoque y alcance}

La Revista de Arquitectura (Bogotá) ( (ISSN 1657-0308 Impresa y E-ISSN 2357-626X en línea) es una publicación científica seriada de acceso abierto, arbitrada mediante revisión por pares (doble ciego) e indexada, en donde se publican resultados de investigación originales e inéditos.

Está dirigida a la comunidad académica y profesional de las áreas afines a la disciplina. Es editada por la Facultad de Diseño y el Centro de Investigaciones (CIFAR) de la Universidad Católica de Colombia en Bogotá (Colombia).

La principal área científica a la que se adscribe la Revista de Arquitectura (Bogotá) según la OCDE es:

Gran área: 6. Humanidades

Área: 6.D. Arte

Disciplina: 6D07. Arquitectura y Urbanismo

También se publican artículos de las disciplinas como 2A02, Ingeniería arquitectónica; 5C03, Estudios urbanos (planificación y desarrollo); 6D07, Diseño.

Los objetivos de la Revista de Arquitectura (Bogotá) son:

- Promover la divulgación y difusión del conocimiento generado a nivel local, nacional e internacional

- Conformar un espacio para la construcción de comunidades académicas y la discusión en torno a las secciones definidas.

- Fomentar la diversidad institucional y geográfica de los autores que participan en la publicación.

- Potenciar la discusión de experiencias e intercambios científicos entre investigadores y profesionales.

- Contribuir a la visión integral de la arquitectura, por medio de la concurrencia y articulación de las secciones mediante la publicación de artículos de calidad.

- Publicar artículos originales e inéditos que han pasado por revisión de pares, para asegurar que se cumplen las normas éticas, de calidad, validez científica, editorial e investigativa.

- Fomentar la divulgación de las investigaciones y actividades desarrolladas en la Universidad Católica de Colombia.
Palabras clave de la Revista de Arquitectura (Bogotá): arquitectura, diseño, educación arquitectónica, proyecto y construcción, urbanismo.

Idiomas de publicación: español, inglés, portugués y francés. Título abreviado: Rev. Arquit.

Titulo corto: RevArq

\section{Políticas de sección}

La revista se estructura en tres secciones correspondientes a las líneas de investigación activas y aprobadas por la institución, y dos complementarias, que presentan dinámicas propias de la Facultad de Diseño y las publicaciones relacionadas con la disciplina.

Cultura y espacio urbano. En esta sección se publican los artículos que se refieren a fenómenos sociales en relación con el espacio urbano, atendiendo aspectos de la historia, el patrimonio cultural y físico, y la estructura formal de las ciudades y el territorio.

Proyecto arquitectónico y urbano. En esta sección se presentan artículos sobre el concepto de proyecto, entendido como elemento que define y orienta las condiciones proyectuales que devienen en los hechos arquitectónicos o urbanos, y la forma como estos se convierten en un proceso de investigación y nuevo de conocimiento. También se presentan proyectos que sean resultados de investigación, los cuales se validan por medio de la ejecución y transformación en obra construida del proceso investigativo. También se contempla la publicación de investigaciones relacionadas con la pedagogía y didáctica de la arquitectura, el urbanismo y el diseño.

Tecnología, medioambiente y sostenibilidad. En esta sección se presentan artículos acerca de sistemas estructurales, materiales y procesos constructivos, medioambiente y gestión, relacionados con los entornos social-cultural, ecológico y económico.

Desde la Facultad. En esta sección se publican artículos generados en la Facultad de Diseño, relacionados con las actividades de docencia, extensión, formación en investigación o internacionalización, las cuales son reflejo de la dinámica y de las actividades realizadas por docentes, estudiantes y egresados; esta sección no puede superar el $20 \%$ del contenido.

Textos. En esta sección se publican reseñas, traducciones y memorias de eventos relacionados con las publicaciones en Arquitectura y Urbanismo.
A Frecuencia de publicación

Desde 1999 y hasta el 2015, la Revista de Arquitectura (Bogotá) publicó un volumen al año, a partir del 2016 se publicarán dos números por año en periodo anticipado, enero-junio y julio-diciembre, pero también maneja la publicación anticipada en línea de los artículos aceptados (versión Post-print del autor).

La Revista de Arquitectura (Bogotá) se divulga mediante versiones digitales (PDF, HTML, EPUB, XML) e impresascon un tiraje de 700 ejemplares, los tiempos de producción de estas versiones dependerán de los cronogramas establecidos por la editorial.

Los tiempos de recepción-revisión-aceptación pueden tardar entre seis y doce meses dependiendo del flujo editorial de cada sección y del proceso de revisión y edición adelantado.

Con el usuario y contraseña asignados, los autores pueden ingresar a la plataforma de gestión editorial y verificar el estado de revisión, edición o publicación del artículo.
A Canje

La Revista de Arquitectura (Bogotá) está interesada en establecer canje con publicaciones académicas, profesionales o científicas del área de Arquitectura y Urbanismo, como medio de reconocimiento y discusión de la producción científica en el campo de acción de la publicación.

Mecanismo

Para establecer canje por favor descargar, diligenciar y enviar el formato: RevArq FP20 Canjes

Universidad Católica de Colombia (2021,
junio-diciembre). Revista de
Arquitectura (Bogotá), 23(2),
I-132. Doi: 10.14718
ISSN: I657-0308
E-ISSN: 2357-626X
Especificaciones:
Formato: $34 \times 24 \mathrm{~cm}$
Papel: Mate II5 g
Tintas: Negro y policromía

A Contacto

Dirección postal:

Avenida Caracas N ${ }^{0} \cdot 46-72$ Universidad Católica de Colombia Bogotá D. C., Colombia Código postal: 111311

Facultad de Diseño Centro de Investigaciones (CIFAR) Sede El Claustro. Bloque "L", 4 piso Diag. 46A N ${ }^{0 .} 15 b-10$ Editor, Arq. César Eligio-Triana

Teléfonos:

+57 (1) $3277300-3277333$

Ext. $3109 ; 3112$ o 5146
Fax: +57 (1) 2858895

Correo electrónico:

revistadearquitectura@ucatolica.edu.co cifar@ucatolica.edu.co

Página WEB:

www.ucatolica.edu.co

Vínculo Revistas científicas

http://publicaciones.ucatolica.edu.co revistas-cientificas

https://revistadearquitectura.ucatolica.edu.co/ 
